\title{
Die Bedeutung von Öffentlichkeit und Privatheit im Medien-/Urheberrecht und in der Medienforschung
}

\section{Tagungsbericht zu dem gleichnamigen Symposium zum UFITA- Relaunch des Instituts für Urheber- und Medienrecht am 29.6.2018 in München}

\section{Armelle Grandjean*}

Das Institut für Urheber- und Medienrecht veranstaltete am 29.6.2018 im Literaturhaus München anlässlich der Neuauflage der UFITA ein Symposium zu dem Thema „Die Bedeutung von Öffentlichkeit und Privatheit im Medien-/Urheberrecht und in der Medienforschung“, welches in interdisziplinären Beiträgen aus urheber- und persönlichkeitsrechtlicher Perspektive ebenso wie aus Sicht der Medienforschung die Begriffe „Öffentlichkeit“ und „Privatheit“" sowie ihr Verhältnis zueinander im Wandel der Zeit untersuchte. ${ }^{* *}$

Nach der Begrüßung und einer kurzen Vorstellung der Neuauflage dieser traditionsreichen medienrechtlichen Archivzeitschrift sowie einer Einführung in die Thematik der Veranstaltung durch Prof. Dr. Nadine Klass, LL.M. (Wellington), Co-Direktorin des Instituts für Urheber- und Medienrecht und Inhaberin des Lehrstuhls für Bürgerliches Recht, Recht des Geistigen Eigentums sowie deutsches und europäisches Verfahrensrecht an der Universität Mannheim, widmete sich Prof. Dr. Birgit Stark, Professorin für Kommunikationswissenschaft mit dem inhaltlichen Schwerpunkt Medienkonvergenz am Institut für Publizistik der Johannes Gutenberg-Universität Mainz und Direktorin des Mainzer Medieninstituts, in ihrem Auftaktvortrag algorithmenbasierten Kommunikationslogiken und ihren Folgen für die Öffentlichkeit. ${ }^{1}$ Daraufhin nahm Prof. Dr. Franz Hofmann, LL.M. (Cambridge), Inhaber des Lehrstuhls für Bürgerliches Recht, Recht des Geistigen Eigentums und Technikrecht an der Friedrich-Alexander-Universität Erlangen-Nürnberg, den Begriff der Öffentlichkeit im Urheberrecht und dabei insbesondere die Konturierung der „öffentlichen Wiedergabe“ durch den $\mathrm{EuGH}^{2}$ in den Blick. ${ }^{3}$ Im Anschluss gab Dr. Bernd Justin Jütte, LL.M. (Luxem-

* Die Verf. ist Rechtsassessorin und Wissenschaftliche Assistentin am Institut für Urheber- und Medienrecht, München.

** Die Durchführung der Veranstaltung wurde durch freundliche Unterstützung der Bayerischen Staatskanzlei gewährleistet.

1 Vortrag nicht veröffentlicht, zur Diskussion um das Konzept der Privatheit/Privatsphäre aus Sicht der Kommunikationswissenschaft vgl. aber den ebenfalls im Zusammenhang mit den Tagungsbeiträgen abgedruckten Aufsatz von Facciorusso, UFITA 2018, 459.

2 Vgl. nur EuGH ZUM 2014, 289 - Svensson; ZUM 2015, 141 - BestWater International; ZUM 2016, 975 - GS Media; ZUM-RD 2017, 309 - AKM/Zürs.net; ZUM 2017, 587 - Filmspeler; ZUM 2017, 746 - The Pirate Bay.

3 Hofmann, UFITA 2018, 334.

UFITA 2/2018, DOI: 10.5771/2568-9185-2018-2-486 
bourg), Research Fellow an der University of Nottingham, einen Überblick über Facetten aus dem europäischen Urheberrecht ${ }^{4}$ bezüglich eines horizontalen Konzepts der Öffentlichkeit. ${ }^{5}$ Schließlich untersuchte Dr. Felix Laurin Stang, LL.M. (Columbia), Rechtsanwalt bei Raue LLP in Berlin, anhand der sogenannten „Pranger“-Entscheidung des OLG München ${ }^{6}$ die Berichterstattung über Social-Media-Inhalte aus urheber- und persönlichkeitsrechtlicher Sicht. ${ }^{7}$

$\mathrm{Zu}$ Beginn der Diskussion stellte Prof. Dr. Klass zusammenfassend fest, dass der Begriff der Öffentlichkeit in zahlreichen Teilbereichen - mit teils unterschiedlichem Verständnis - eine Rolle spielt, was die Frage nach der Existenz von Teilöffentlichkeiten und der Notwendigkeit, diese im Recht abzubilden, aufwerfe. Unter ihrer Moderation bestand nun die Möglichkeit zu Fragen und Anmerkungen aus dem Publikum. Patricia Biondo, CrossCultural Communication Consulting in München, wollte daraufhin wissen, ob die Differenzierung zwischen den Kategorien „Freunde“ und „öffentlich“ auf Facebook überhaupt eine Relevanz habe, was Dr. Stang bejahte. Er verwies in diesem Zusammenhang auf weitere mögliche Voreinstellungen des Profils, wie etwa die Auffindbarkeit durch Suchmaschinen, und machte ferner deutlich, dass im Persönlichkeitsrecht aufgrund der dogmatischen Konstruktion der Abwägung auch der Grad der Öffentlichkeit berücksichtigt werden könne. Dr. Stang wies jedoch zugleich darauf hin, dass eine Rechtsverletzung, wie etwa eine Verleumdung, auch im „Freundeskreis“ stets justiziabel sei. Prof. Dr. Hofmann ergänzte hierzu aus urheberrechtlicher Sicht, dass sich hier auch die Frage einer öffentlichen Wiedergabe stelle. So könne im Einzelfall je nach Anzahl und Art der Freunde selbst der Freundeskreis öffentlich im urheberrechtlichen Sinne sein.

Prof. Dr. Jan Oster, LL.M. (Berkeley), Universität Leiden, machte im Anschluss gegenüber Dr. Stang, der unter anderem berichtet hatte, dass der EGMR regelmäßig die Funktion der journalistischen Medien als „Public Watchdog“ hervorhebe, deutlich, dass er dies nur für die halbe Wahrheit halte, da der EGMR zugleich auch die Pflichten und Verantwortungen dieser Medien betone. So habe etwa in dem von Prof. Dr. Stark in ihrem Vortrag angeführten Fall $^{8}$ ein journalistisches Medium mit der traditionellen Gatekeeper-Funktion die Klägerin gewissermaßen auf ein Podium, also an den Pranger gestellt. Daher sei er nicht sicher, ob der EGMR sich hier nicht sogar gegebenenfalls auf die Seite der Facebook-Nutzerin schlagen und Art. 8 EMRK als gegenüber Art. 10 EMRK vorrangig ansehen würde. Dr. Stang gestand insofern zu, dass der Fall vielfältige Probleme aufwerfe, denen man sich auf verschiedene Weise nähern könne, und betonte insbesondere zwei Aspekte: Zum einen sei der Begriff des „Prangers“ in der Berichterstattung nicht deckungsgleich mit dem

4 Richtlinie 2001/29/EG des Europäischen Parlaments und des Rates v. 22.5.2001 zur Harmonisierung bestimmter Aspekte des Urheberrechts und der verwandten Schutzrechte in der Informationsgesellschaft, AB1. Nr. L 167 v. 22.6.2001, S. 10-19.

5 Jütte, UFITA 2018, 354.

6 OLG München ZUM-RD 2018, 402.

7 Stang, UFITA 2018, 375.

8 LG Saarbrücken ZUM-RD 2018, 115 m. Anm. Ladeur. 
gleichnamigen von der Rechtsprechung entwickelten rechtlichen Begriff. Bei letzterem gehe es im Ergebnis, auch wenn eine nähere Definition fehle, um eine Art hilflosen Ausgeliefertseins gegenüber der Berichterstattung. Das Entscheidende mit Blick auf die Rechtsprechung des Bundesverfassungsgerichts sei, dass derjenige die Regeln des Meinungskampfes festlege, der sich als erster äußere. Wenn dieser betreffend sowohl den Inhalt der Nachricht als auch die Art der Aussage gleichsam mit offenem Visier eine extreme Ausdrucksweise wähle, müsse sich aus seiner Sicht auch das Medium nicht zurückhalten. Bezüglich der Frage der Erwartbarkeit ist aus Sicht von Dr. Stang nur langsam ein gewisser Bewusstseinswandel festzustellen. So seien sich die bei Facebook postenden Nutzer oft nicht über den Verbreitungsgrad ihrer Posts bewusst.

Daraufhin wandte sich Prof. Dr. Michael Grünberger, LL.M. (NYU), Direktor des Instituts für Urheber- und Medienrecht und Inhaber des Lehrstuhls für Bürgerliches Recht, Wirtschafts- und Technikrecht der Universität Bayreuth, an Prof. Dr. Stark. Er betonte nochmals die in ihrem Vortrag aufgezeigte Besonderheit, dass ein klassisches Medium, welches bestimmten Regeln unterliege, plötzlich die Logik eines anderen Mediums fast schon usurpiere und dadurch eine völlig neue Wirkung erziele. Angesichts der von ihr beschriebenen Einflüsse von Intermediären auf die klassischen Medien wollte er wissen, wie sich dieser Wandel konkret auswirke, über welche Erfahrungen die Medienwissenschaft hier verfüge und welche Handlungsanleitungen normativer Art sie zur Diskussion stellen würde. Prof. Dr. Stark ergänzte zunächst, dass auch sie der Ansicht sei, dass sich die User oft nicht der Folgen ihres Handelns und der damit generierten Art von Öffentlichkeit bewusst seien. Sie sprach in diesem Zusammenhang von einem unbedarften, geradezu naiven Verhalten. Durch die digitalen Angebote der traditionellen Medien finde eine Erweiterung der Öffentlichkeit statt. In dem von Dr. Stang geschilderten Fall ${ }^{9}$ hätte zudem eine ganz bewusste Provokation seitens der Boulevardzeitung vorgelegen, um die User an den Pranger zu stellen. Man könne dies nicht mit anderen Medien, insbesondere mit Qualitätszeitungen vergleichen, die sich selbst ganz andere Regeln auferlegten. Sie führte daher Redaktionsstatuten als mögliches Mittel zur Verhinderung solcher Äußerungen für die Zukunft an. Dr. Stang ergänzte hierzu, dass auch durchaus als seriös eingestufte Medien teilweise mit Einblendung des Namens und Profilbildes über entsprechende Posts berichten würden, wohingegen andere Medien anonymisieren würden. Er ließ durchblicken, dass es sehr interessant sei, was sich hier in der Zukunft für journalistische Ehrenkodizes etablieren würden, wofür er nochmals die Bedeutung des konkreten Falls als möglichen Präzedenzfall betonte.

Prof. Dr. Klass wollte daran anschließend von Prof. Dr. Stark wissen, ob aus juristischer Sicht bei der rechtlichen Regulierung etwas stärker in den Blick genommen werden müsse, dass insbesondere für jüngere Nutzer die Teilnahme an einem solchen Millionennetzwerk und damit eine gewisse Selbstöffnung integrierend erforderlich sei, um nicht auch im analogen Bereich in gewisser Weise einer Ausgrenzung zu unterliegen. Sie warf daher die Frage auf, ob insofern möglicherweise ein Umdenken im Sinne des Überdenkens der etablierten 
Sphärentheorie angezeigt sei, also die Aufgabe der strengen Differenzierung zwischen öffentlich und privat, um geschützte Räume auch im öffentlichen Bereich zu schaffen, in denen Persönlichkeitsentwickelung und Kommunikation ermöglicht werden. Prof. Dr. Stark erwiderte hierauf, dass die Idee geschützter Räume im öffentlichen Bereich zwar begrüßenswert, aber nicht realistisch sei. So basiere die gesamte Logik des Netzwerkes Facebook auf einer gewissen Selbstöffnung der Menschen, weshalb eine Einschränkung an dieser Stelle der Funktionsweise der Plattform diametral entgegenstehe.

Prof. Dr. Grünberger nahm daraufhin nochmals auf drei bereits von Prof. Dr. Stark aufgeworfene Punkte Bezug: Zum ersten hatte sie die Auswahlkriterien von Algorithmen gesteuerter Plattformen angesprochen, die filtern, sortieren und personalisieren, woraus er die Frage ableitete, ob Twitter hier eine Sonderrolle zukomme. Der zweite Aspekt betreffe insbesondere Juristen in transnationalen Diskursen: Seiner Ansicht nach gebe es entgegen der von Prof. Dr. Stark vertretenen These sehr wohl eine nationale Bindung an eine bestimmte Rechtsordnung, nämlich in Bezug auf den Standort des Geschäftssitzes. Er verwies hierzu speziell auf Kalifornien und das dort vorherrschende Verständnis sowie auf das Thema der Content-Regulierung bei sozialen Netzwerken, wo etwa ein Spannungsverhältnis zwischen Sexualität und Free Speech herrsche. Schließlich kam Prof. Dr. Grünberger auch nochmals auf das von Prof. Dr. Stark monierte Fehlen ethischer Grundlagen bei den neuen Medien zurück. Er verwies auf die mit dem stark umstrittenen NetzDG ${ }^{10}$ nun vorliegende rechtliche Regulierung, die teilweise damit gerechtfertigt wurde, dass die Selbstregulierung der in Frage stehenden sozialen Systeme bislang nicht funktioniere oder nicht existiere. Gerade im journalistischen Bereich seien aber möglicherweise weniger Regelungen angebracht Art. 1 GG gewähre hier einen sehr großen Freibereich -, da man auf die Eigenrationalität und damit die Selbstregulierung der Kommunikationsmedien vertrauen könne. Wenn jedoch Normen in sozialen Kontexten fehlten, müssten Juristen sie in Erfüllung der klassischen Aufgabe des Rechts konstruieren. Von daher sieht Prof. Dr. Grünberger hier tatsächlich Bedarf für eine Form von rechtlicher Regulierung, wobei er offenließ, ob diese konkret so ausgestaltet sein muss wie das NetzDG.

Prof. Dr. Stark erwiderte hierauf zunächst, dass Twitter in der Tat insofern eine Sonderrolle einnehme, als es nicht in der breiten Bevölkerung Anwendung finde, sondern lediglich bei 3 bis $4 \%$ der Internetnutzer. Sie erläuterte, dass sich in der Kommunikationswissenschaft mittlerweile die Sichtweise etabliert habe, dass Twitter ein spezielles Forum für Journalisten und Medienfachleute sei und eine ganz eigene Öffentlichkeit konstruiere, von der oft die Initialzündung in dem dynamischen Prozess des Veröffentlichens ausgehe. Zu dem zweiten von Prof. Dr. Grünberger angeführten Punkt bezüglich einer nationalen Bindung gestand sie zu, dass es geschäftsmäßig eine solche durchaus geben möge. Sie habe es dagegen eher im Vergleich dazu gemeint, wie sich traditionelle Medien mit einer bestimmten kulturellen Prägung in einem Land etablieren, und verwies hierzu beispielshalber auf in

10 Gesetz zur Verbesserung der Rechtsdurchsetzung in sozialen Netzwerken (Netzwerkdurchsetzungsgesetz - NetzDG) vom 1. September 2017, BGB1. I, S. 3352-3355. 
Österreich existierende Gratismedien mit eigens etablierten Normen. Die nationale Bindung spiele dabei auch für die Art der Berichterstattung, was in der Boulevardberichterstattung möglich sei, eine Rolle. Eine in diesem Sinne verstandene nationale Bindung fehle eben bei der Plattform. Schließlich entgegnete sie zu dem Aspekt des Fehlens ethischer Grundlagen, dass man von daher aus ihrer Sicht durchaus die Forderung nach einer Art der Regulierung stellen könne, wobei verschiedene Formen diskutiert würden: Klassische Selbstregulierung, die beispielsweise Google insbesondere im Bereich des Jugendschutzes für sich in Anspruch nehme, habe jedoch bislang versagt, da der Nutzer diese Informationen im Netz nicht finde und auch nicht aktiv danach suche. Deshalb würden nun verstärkt Ko-Regulierungsmaßnahmen gefordert, also Regulierung auf mehreren Ebenen einschließlich staatlicher Überwachung, Kontrolle und Sanktion.

Prof. Dr. Mark D. Cole, Professor für Medien- und Telekommunikationsrecht an der Universität Luxemburg und Wissenschaftlicher Direktor des Instituts für Europäisches Medienrecht (EMR) in Saarbrücken, kam daraufhin auf Dr. Stang zurück, der gesagt hatte, dass Überlegungen zur Anwendbarkeit urheberrechtlicher Grundsätze auf das Persönlichkeitsrecht noch nicht sehr weit fortgeschritten seien, und verwies auf die „Deckmyn“-Entscheidung des EuGH, ${ }^{11}$ in der seiner Ansicht nach zumindest ein Versuch in diese Richtung gemacht worden sei. So könne man durchaus überlegen, ob die Grenze der Satire/Parodie im Urheberrecht, wo auch das Urheberpersönlichkeitsrecht eine Rolle spiele, ein geeigneter Weg sei, um bei dem Inverkehrbringen eines urheberrechtlich geschützten Werkes in die Öffentlichkeit diese zweite Komponente der Verletzung des Ansehens des Urhebers anzunehmen. Von Dr. Jütte und Prof. Dr. Hofmann wollte er daher wissen, ob sie eine Möglichkeit sähen, diese Brücke zwischen den beiden bislang getrennt betrachteten Rechtsbereichen zu schlagen. Dr. Jütte erwiderte hierauf, dass der EuGH sich auch in der „Deckmyn“Entscheidung nicht direkt damit befasst habe, sondern zu einer Lösung des Falles vielmehr über die Anwendung des Gleichbehandlungsgrundsatzes gekommen sei, verwies aber auf ein weiteres vor dem EuGH anhängiges Verfahren, ${ }^{12}$ in dem dieser möglicherweise Anlass zu einer Darstellung habe, wie diese Problematik auf europarechtlicher Ebene gelöst werden könne. Prof. Dr. Hofmann ergänzte, dass der Öffentlichkeitsbezug zwar Teil des mit dem Urheberrecht verfolgten Interessenausgleichs sei, eine direkte Übertragung aus seiner Sicht aber mit Vorsicht zu genießen und im jeweiligen Kontext zu behandeln sei.

Im Anschluss warf Margherita Checchin, Rechtsanwältin im Justiziariat des WDR in Köln, bezogen auf die Frage der Regulierung noch einmal den aus ihrer Sicht zu kurz gekommenen Aspekt des Zugangs zur Öffentlichkeit in den Raum, der aus ihrer Sicht in der digitalen Welt nicht zwingend erleichtert sei. Prof. Dr. Stark entgegnete dem, dass es im Vergleich zur analogen Welt durchaus einen technisch erweiterten Zugang gebe, öffentlich zu diskutieren, etwa durch Plattformen wie Facebook. Zugleich gestand sie zu, dass man sich bei Inanspruchnahme dieser Möglichkeit auch entsprechenden Regeln unterwerfen

11 EuGH ZUM-RD 2014, 613 - Deckmyn.

12 BGH ZUM-RD 2017, 581 - Reformistischer Aufbruch. 
müsse, was nicht nur für Privatpersonen, sondern insbesondere auch für Medienunternehmen gelte. Anderenfalls könne diese Art der Kreation einer Öffentlichkeit nicht funktionieren. Prof. Dr. Hofmann machte noch darauf aufmerksam, dass man jedes Problem genau zuordnen müsse und es keine „Weltformel“, auch nicht etwa des urheberrechtlichen Öffentlichkeitsbegriffs, gerade für die Frage des Spannungsfeldes zwischen Zugangsregel und Kommunikationsfreiheit gebe.

Monika Ermert, freie Journalistin/eLance journalist aus München, meldete sich daran anschließend mit der Frage, ob nicht durch das NetzDG die Kommunikationsfreiheit gerade wieder eingeschränkt und die Deutungsmacht in die Hände der Privaten gegeben werde. Sie wollte daher wissen, wie eine Regulierung aussehen könne, ohne diese Einschränkungen in Kauf nehmen zu müssen. Prof. Dr. Hofmann verwies zunächst auf den allgemeinen Grundsatz, dass das Recht auch im Internet durchzusetzen sei, weshalb etwa auch mittelbar Verantwortliche (wie Plattformbetreiber) unter gewissen Voraussetzungen haften. Vor dem Hintergrund dieses allgemeinen Rechtsgedankens halte er das NetzDG dem Grundsatz nach für richtig. Er machte deutlich, dass es in einem ersten Schritt gelungen sei, durch die Störerhaftung, die europäische Intermediärshaftung und nun das Recht der öffentlichen Wiedergabe zu verhindern, dass das Internet ein rechtsfreier Raum ist, indem rechtswidrige Inhalte entfernt werden müssen. Nun müsse jedoch in einem zweiten Schritt dafür gesorgt werden, dass zu Unrecht gesperrte Inhalte (etwa eine im Nachhinein als zulässig zu bewertende Meinungsäußerung auf Facebook) wieder zurück in die Öffentlichkeit gelangen. Er erläuterte, dass hier verschiedene Ansatzpunkte, etwa über die Grundrechte oder aber auch durch Vertrag, möglich seien.

Nach einer Mittagspause führte Prof. Dr. Grünberger in den zweiten Teil der Veranstaltung ein, in dem zunächst Prof. Dr. Anne Lauber-Rönsberg, Inhaberin der Juniorprofessur für Bürgerliches Recht, Immaterialgüterrecht, insbesondere Urheberrecht, sowie Medienund Datenschutzrecht am Institut für Geistiges Eigentum, Wettbewerbs- und Medienrecht der Technischen Universität Dresden, die Auswirkungen der DSGVO ${ }^{13}$ auf das zivilrechtliche Äußerungsrecht aufzeigte. ${ }^{14}$ Dr. Christian Nuernbergk, Inhaber einer Vertretungsprofessor für Medien- und Kommunikationswissenschaft an der Universität Trier und Wissenschaftlicher Mitarbeiter am Institut für Kommunikationswissenschaft und Medienforschung der Ludwig-Maximilians-Universität München, referierte daraufhin über Öffentlichkeit und mediale Partizipation unter digitalen Vorzeichen. ${ }^{15}$ Zum Abschluss sprach Prof. Dr. Mark D. Cole über das Zielpublikum „Öffentlichkeit“ als Anknüpfungspunkt für (Medien-)Regulierung. ${ }^{16}$

13 Verordnung (EU) 2016/679 des Europäischen Parlaments und des Rates vom 27. April 2016 zum Schutz natürlicher Personen bei der Verarbeitung personenbezogener Daten, zum freien Datenverkehr und zur Aufhebung der Richtlinie 95/46/EG (Datenschutz-Grundverordnung), ABl. Nr. L 119 v. 4.5.2016, S. $1-88$.

14 Lauber-Rönsberg, UFITA 2018, 398.

15 Vortrag nicht veröffentlicht.

16 Cole, UFITA 2018, 436. 
Anschließend bestand wiederum die Möglichkeit zu Fragen und Anmerkungen aus dem Publikum. Prof. Dr. Hofmann zeigte sich zunächst überzeugt von den Ausführungen, was alles unter den Begriff der Öffentlichkeit fällt, warf aber die Frage nach dem Nutzen dieser Subsumtion auf, wenn dann doch wieder differenziert reguliert werden müsse, wie etwa im Urheberrecht mithilfe von Schrankenregelungen. Prof. Dr. Cole gab Prof. Dr. Hofmann diesbezüglich im Ergebnis Recht: Die Medienpolitik oder der Regulierer werde sich dann überlegen müssen, wie es in der Praxis funktionieren könne, wofür derselbe Gedankenaufwand nötig sei wie bei einer rein sektoriellen Regulierung. Insofern sei aus ökonomischer Sicht (etwa durch Beschleunigung oder Vereinfachung der Vorgänge) kein Gewinn ersichtlich. Er sprach jedoch in diesem Zusammenhang von einer seiner Ansicht nach wichtigen Wahrnehmungsverschiebung: Wer im gleichen Feld dem Grundsatz nach reguliert werde, sei in einem Level Playing Field, weshalb seiner Ansicht nach auch neue Inhalteverbreitungsformen zunächst einmal unter den Grundansatz möglicher Regulierung gefasst werden sollten. In einem zweiten Schritt könne man sich dann Gedanken über die konkrete Ausgestaltung der Regulierung machen. Als Gegenbeispiel verwies er auf das Haftungsprivileg der E-Commerce-RL, ${ }^{17}$ welches nun punktuell angepasst wird, wohingegen er für eine vollständige Abschaffung als ,ehrlichere“ Lösung plädiere, die dann immer noch eine anschließende Differenzierung erlaube.

Prof. Dr. Klass wollte daraufhin von Dr. Nuernbergk wissen, ob er auch untersuche, welche Rolle die bei digitalen Angeboten sehr viel stärker vorhandene Anonymität der Nutzer spiele, und wie sich diese auf den Meinungsbildungsprozess auswirke. Dr. Nuernbergk gestand zu, dass die Anonymität bei Phänomenen wie den Nutzerkommentaren, also bei der medialen Partizipation, ein wichtiger Aspekt sei. Während Plattformen zunächst unterschiedliche Grade von Anonymität bei der Kommentarfunktion zugelassen hätten mit der entsprechenden Folge unterschiedlicher Beteiligung, habe sich dies jedoch mittlerweile angeglichen. Von der anderen Seite betrachtet rückten durch die Anonymität identitätsbezogene Merkmale bezogen auf die Person in den Hintergrund, wohingegen solche bezogen auf die Gruppenidentität im Vordergrund stünden. Dies habe dann auch Auswirkungen auf die Art und Weise der Kommunikation, indem es zu einer Verschärfung des Kommunikationsstils führe, so Dr. Nuernbergk.

Prof. Dr. Grünberger wandte sich im Anschluss mit der Frage an Prof. Dr. LauberRönsberg, wie angesichts dessen, dass wir auf der einen Seite das einheitliche Regime der DSGVO und auf der anderen Seite ihrer Auffassung nach weiterhin disparate Regime im Äußerungsrecht hätten, mit den Gerichtsstandsmöglichkeiten aus Art. 7 der Brüssel-Ia-

17 Richtlinie 2000/31/EG des Europäischen Parlaments und des Rates vom 8. Juni 2000 über bestimmte rechtliche Aspekte der Dienste der Informationsgesellschaft, insbesondere des elektronischen Geschäftsverkehrs, im Binnenmarkt („Richtlinie über den elektronischen Geschäftsverkehr“), AB1. Nr. L 178 v. 17.7.2000, S. 1-16. 
$\mathrm{VO}^{18}$ umzugehen sei, der es ermögliche, unterschiedliche Haftungsregime auf verschiedene Sachverhalte anzuwenden. Prof. Dr. Lauber-Rönsberg verwies zunächst auf den Hinweis in Erwägungsgrund 153 der DSGVO bezüglich des anwendbaren Rechts. Dem begegnete Prof. Dr. Grünberger wiederum mit dem Verweis auf die Rom-II-VO, ${ }^{19}$ die sämtliche Persönlichkeitsrechte ausdrücklich aus ihrem Anwendungsbereich ausschließt. Er machte deutlich, dass er es für problematisch halte, über einen Erwägungsgrund eine kollisionsrechtliche Regelung zu treffen in einem Bereich, in dem sich die Mitgliedstaaten die Kompetenz nicht aus der Hand nehmen ließen. Dies gestand auch Prof. Dr. Lauber-Rönsberg zu, zumal die Erwägungsgründe von ihrer interpretatorischen Aussagekraft durchaus sehr begrenzt seien, da sie zum Teil auch gar nicht an die letzte Fassung der DSGVO angepasst wurden. Von daher müsse ihrer Ansicht nach genau nachvollzogen werden, aus welchem Stadium der entsprechende Erwägungsgrund stamme und ob es zu diesem Zeitpunkt möglicherweise andere Regelungen in der DSGVO gab.

Prof. Dr. Oster warf daran anknüpfend die Frage auf, inwiefern die Gefahr bestehe, dass das Datenschutzrecht auf Dauer zu einer Art Ersatzdeliktsrecht werde, was dazu führe, dass die Brüsel-Ia-VO und Rom-II-VO obsolet werden und das ausdifferenzierte allgemeine Persönlichkeitsrecht überholt. Prof. Dr. Lauber-Rönsberg machte deutlich, dass es von der jeweiligen Perspektive abhänge, ob man hierin eine Gefahr oder aber eine Chance für den Datenschutz sehe. Zudem erläuterte sie ergänzend zu ihrem Vortrag, dass die Tatsache der Anwendbarkeit des Datenschutzrechts noch nicht bedeute, dass Berichterstattungen grundsätzlich unzulässig seien. Sie verwies hierzu auf die Erlaubnistatbestände in Art. 6 Abs. 1 DSGVO, die wesentlich auslegungsoffener seien als im bisherigen Datenschutzrecht. Auch bei den Betroffenenrechten könne man Einschränkungen in die Regelungen hineininterpretieren. Schließlich verwies sie auf die Öffnungsklauseln, die weitere Einschränkungen zulassen. Die eigentliche Fragestellung sei ihrer Ansicht nach, welches das geeignete Rechtsregime sei, um hier das Recht der informationellen Selbstbestimmung und die Kommunikationsfreiheiten im Sinne einer praktischen Konkordanz in Einklang zu bringen. Die bisherige im Äußerungsrecht bestehende deutsche Lösung berge zwar die Gefahr, dass diese Abwägungen zum Teil - je nachdem, wie weit die Öffnungsklauseln (auch durch den EuGH) interpretiert werden - in das Datenschutzrecht hineingezogen würden. So habe sie im Moment den Eindruck, dass durch den EGMR, dessen Rechtsprechung auch durch den EuGH zu berücksichtigen ist, die Kommunikationsfreiheiten eine sehr starke Stellung haben. Hier stelle sich dann aber insbesondere die Frage der behördlichen Durchsetzung, die sie für nicht adäquat halte für diesen Bereich, weshalb sie den Weg über das Äußerungsrecht bevorzuge. Eine andere Frage sei, dass durch die jahrzehntelange Entwicklung

18 Verordnung (EU) Nr. 1215/2012 des Europäischen Parlaments und des Rates vom 12. Dezember 2012 über die gerichtliche Zuständigkeit und die Anerkennung und Vollstreckung von Entscheidungen in Zivil- und Handelssachen, AB1. Nr. L 351 vom 20.12.2012, S. 1-32.

19 Verordnung (EG) Nr. 864/2007 des Europäischen Parlaments und des Rates vom 11. Juli 2007 über das auf außervertragliche Schuldverhältnisse anzuwendende Recht („Rom II“), ABl. Nr. L 199 vom 31.7.2007, S. 40-49. 
bestimmter Fallgruppen die Rechtssicherheit nun etwas leiden könne, was aber in diesem Fall in Kauf zu nehmen sei, so Prof. Dr. Lauber-Rönsberg.

Frank Michael Höfinger, Rechtsanwalt bei Lausen Rechtsanwälte in Köln, warf diesbezüglich daraufhin mit den Informationspflichten nach der DSGVO ein weiteres praktisches Problem auf. So zeige sich aus seiner Sicht bei der Beratung betreffend insbesondere Bildaufnahmen für nicht journalistisch-redaktionelle Zwecke, etwa aus PR-Gründen, ganz deutlich, dass es nicht adäquat sei, dort die DSGVO anzuwenden. Prof. Dr. Lauber-Rönsberg gab zu, dass dies stark umstritten sei. Wenn dieser Fall tatsächlich unter der DSGVO zu lösen sei, stelle sich zum einen die Frage nach Erlaubnistatbeständen (Einwilligung oder Art. 6, etwa die berechtigten Interessen des Verantwortlichen) und zum anderen bestünden Informationspflichten sowie weitere Betroffenenrechte. Sie verwies hierbei auf die beiden Stellungnahmen der Brandenburgischen ${ }^{20}$ und des Hamburgischen Landesdatenschutzbeauftragten, ${ }^{21}$ die Überlegungen dazu enthalten, anstelle von Art. 13, der die Informationspflichten bei Datenerhebung von der betroffenen Person selbst beinhaltet, Art. 14 anzuwenden, wenn eine Personengruppe fotografiert wird, die jedenfalls so groß ist, dass es unzumutbar wäre, von jedem Einzelnen eine entsprechende Einwilligung einzuholen. Prof. Dr. Lauber-Rönsberg erläuterte hierzu, dass Art. 14 insoweit einen etwas weniger restriktiven Rechtsrahmen aufweise, als er in Abs. 5 Buchst. b) die Anwendung der Informationspflichten bei Unmöglichkeit oder unverhältnismäßigem Aufwand der Erteilung dieser Informationen ausschließt. Dies sei daher ein möglicher Ansatzpunkt für den deutschen Gesetzgeber, im Rahmen der Öffnungsklausel (Art. 23 DSGVO) die Informationspflichten über die bisherigen Einschränkungen im Bundesdatenschutzrecht hinaus noch weiter einzuschränken, beispielsweise bei letztlich öffentlich zugänglichen Informationen (etwa im Rahmen von öffentlichen Veranstaltungen). Angesichts der Rechtsprechung des EuGH, seine Kompetenzen kontinuierlich zu erweitern, sei jedoch zu erwarten, dass er die Öffnungsklausel eher restriktiv auslegen werde und den Bereich der nicht journalistischen Nutzung beispielsweise in den Zuständigkeitsbereich des europäischen Datenschutzes ziehe.

Prof. Dr. Grünberger kam abschließend noch einmal auf die Aussage von Dr. Nuernbergk, dass die Freiheit des Zugangs zur Öffentlichkeit durch die digitalisierten Foren eingeschränkt werde, sowie auf den von ihm geprägten Begriff der Semi-Öffentlichkeit zurück. Die Vorträge von Prof. Dr. Lauber-Rönsberg und Prof. Dr. Cole hätten diesbezüglich gezeigt, dass es darum gehe, diese Privatisierung von Öffentlichkeit wieder in das Licht der regulierenden Öffentlichkeit zurückzuholen und mit anderen Interessen, auch Datenschutzinteressen, zu beleuchten und zu kontrastieren. Mit herzlichem Dank an die Referenten und Teilnehmer beendete er schließlich die Veranstaltung.

20 Abrufbar unter https://www.lda.brandenburg.de/media_fast/4055/RechtlicheAnforderungen Fotografie.pdf, zuletzt abgerufen am 24.9.2018.

21 Abrufbar unter https://www.privatfunk.de/anl/18_10153_01.pdf, zuletzt abgerufen am 24.9.2018. 